\title{
BIRATIONAL AUTOMORPHISMS OF A THREE-DIMENSIONAL DOUBLE QUADRIC WITH AN ELEMENTARY SINGULARITY
}

\author{
M. M. GRINENKO \\ $17 / \mathrm{FEB} / 97$
}

\begin{abstract}
It is proved that the group of birational automorphisms of a threedimensional double quadric with a singular point arising from a double point on the branch divisor is a semidirect product of the free group generated by birational involutions of a special form and the group of regular automorphisms. The proof is based on the method of 'untwisting' maximal singularities of linear systems.

Bibliography: 5 titles.
\end{abstract}

Preliminary remarks. In this article we study the group of birational automorphisms of a double quadric with a singular point arising from a double point on the branch divisor (cut out by a fourth-degree hypersurface in $\mathbb{P}^{4}$ ). The smooth case was investigated in [1].

It is difficult to say a priori how a double point affects the group $\operatorname{Bir}(V)$ of birational automorphisms of a variety $V$. For example, if $V$ is a three-dimensional smooth quartic, then, as is well known [2], $\operatorname{Bir}(V)$ coincides with the group of automorphisms; however, this is no longer so for a quartic with a double point [3]. On the other hand, double spaces that are smooth or have double points do not have non-trivial birational automorphisms (in all higher dimensions) [4]. In our case (an exact formulation is given below) it turns out, as in the case of a double space, that a singular point does not add anything new to the group $\operatorname{Bir}(V)$ (though it is itself non-trivial).

All our arguments fit in the framework of the method of maximal singularities. The first rigorous exposition of this method is due to Iskovskikh and Manin [2]. Recently the method was essentially reworked by Pukhlikov [5], and this has made it possible to give the arguments a more geometric and explicit character and extend the area of application while avoiding the 'test class' technique. Our article also has the purpose of applying the new technique to the study of varieties with singularities.

1991 Mathematics Subject Classification. Primary 14E07, 14J20; Secondary 14C20, 14E09, 14E05, 14J28, 14J45.

This work was carried out with the financial support of the Russian Foundation for Basic Research (grant no. 96-01-00820) and a grant for Support of Leading Scientific Schools (no. 96-1596146).

English translation: H.H.McFaden

Typeset by $\mathcal{A M}_{\mathcal{M}} \mathcal{S}-\mathrm{T}_{\mathrm{E}} \mathrm{X}$ 
The author would like to express his gratitude to A. V. Pukhlikov for the statement of the problem and for very useful discussions, and to V. A. Iskovskikh for his support and his attention to this work.

\section{$\S 1$. Formulation of the main Result}

We shall be in the following situation everywhere below. Let $F$ be a quartic in $\mathbb{P}^{4}$ that is smooth everywhere except at the point $P$, where it has an ordinary double singularity. Assume that the smooth quadric $Q \subset \mathbb{P}^{4}$ is such that the divisor $F_{Q}=Q \cap F$ passes through $P$, has there an ordinary double singularity, and does not have other singular points. We consider a double cover morphism $\pi: V \rightarrow Q$ that is branched over $F_{Q}$. The variety $V$ has an ordinary double point, which we also denote by $P$, and does not contain other singularities.

We assume in addition that there are precisely 12 lines on $Q$, each passing through $P$ and tangent to $F_{Q}$ at some other point different from $P$, that there are no lines on $Q$ intersecting $F_{Q}$ only at $P$, and that $F$ and $Q$ do not have common lines passing through $P$. These conditions are obviously satisfied for the general quartic $F$.

It is not hard to see that $\operatorname{Pic}(V)=\mathbb{Z}[H]$ for a double quadric $V$, where $H$ is the inverse image of a hyperplane in $\mathbb{P}^{4}$. In fact, more is true: $\mathrm{Cl}(V)=\operatorname{Pic}(V)$, that is, every Weil divisor is linearly equivalent to a Cartier divisor. Indeed, suppose that $\varphi: \widetilde{\mathbb{P}}^{4} \rightarrow \mathbb{P}^{4}$ is the composition of a double cover of $\mathbb{P}^{4}$ with a branch over the quartic $F$ and a subsequent blow-up of the singular point, and let $\widetilde{V}$ and $\widetilde{F}$ be the proper smooth inverse images of $V$ and $F$ in $\widetilde{\mathbb{P}}^{4}$. Then $\widetilde{F}=\varphi^{*}(4 H)-2 E$ and $\widetilde{V}=\varphi^{*}(2 H)-E$, where $E$ is the exceptional divisor of the blow-up, and this implies that $\widetilde{V}$ and $\widetilde{F}$ are ample. Moreover, $\operatorname{Pic}(\widetilde{F})=\mathbb{Z} \oplus \mathbb{Z}$. Using the Lefschetz theorem on hyperplane sections twice, we find that $\mathrm{Cl}(\widetilde{V})=\mathbb{Z} \oplus \mathbb{Z}$, from which we get that $\mathrm{Cl}(V)=\mathbb{Z}$.

Let $B \subset V$ be a smooth curve such that $\left.\pi\right|_{B}: B \rightarrow l$ is an isomorphism onto a line $l \subset Q$. Then $l$ is one of the following three types:

A. $l$ is tangent to $Q_{F}$ at two smooth points;

B. $l$ passes through $P$ and is tangent to $Q_{F}$ at another point;

C. $l \subset Q_{F}$.

The corresponding curves $B$ will be called lines of type A, B, or $\mathrm{C}$ on $V$.

As follows from arguments in [1], there is on $V$ an exactly one-dimensional family of lines with each line intersecting only finitely many others.

Construction of a birational automorphism associated with lines of type A or B on $\boldsymbol{V}$. Let $B \subset V$ be such a line. Then there exists a line $B^{*} \subset V$ conjugate to $B$ with respect to the involution $\delta: V \rightarrow V$ transposing the sheets of the cover $\pi: V \rightarrow Q$. We note that $B$ and $B^{*}$ intersect at two points. The linear system $\left|-K_{V}-B-B^{*}\right|$ gives a rational map $g: V \rightarrow \mathbb{P}^{2}$ that lifts to a morphism $\bar{g}: \bar{V} \rightarrow \mathbb{P}^{2}$, where $\psi: \bar{V} \rightarrow V$ is the composition of a blow-up of the line $B$ and then of the proper inverse image of the line $B^{*}$. We denote by $E$ and $E^{*}$ the corresponding exceptional divisors on $\bar{V}$.

We remark that the general fibre $X$ of the morphism $\bar{g}$ is an elliptic curve that intersects $E^{*}$ in a point. The reflection with respect to this point (in the sense of the group law) gives an automorphism of $X$. This determines on some open subset 
of $\bar{V}$ a regular automorphism that lowers to $V$ as a birational automorphism $\tau_{B}$ (for details on this see [1]).

Birational rigidity. To determine the birational type of $V$ the following definition is useful (see [5]).

Definition. A pair $(W, Y)$, where $W$ is a projective variety that is non-singular in codimension 1 and $Y$ is a (Weil) divisor on $W$, is called a test pair if the following conditions hold:

a) the linear system $|Y|$ is unfixed;

b) there exists a number $\alpha=\alpha(W, Y) \in \mathbb{R}_{+}$such that for every $\beta>\alpha$ with $\beta \in \mathbb{Q}$ the linear system

$$
\left|m\left(Y+\beta K_{W}\right)\right|
$$

is empty for any $m \in \mathbb{Z}_{+}$with $m \beta \in \mathbb{Z}$.

The indicated number $\alpha(W, Y)$ is called the canonical threshold of the pair.

The following are examples of test pairs important in practice:

- $\left(\mathbb{P}^{n}, L\right)$, where $L$ is a hyperplane and here $\alpha\left(\mathbb{P}^{n}, L\right)=1 /(n+1)$;

- the pair $(W, Y)$, where $\varphi: W \rightarrow S$ is a fibring into Fano varieties and $Y$ is the inverse image of a very ample divisor on $S$, and here the canonical threshold is equal to zero;

- finally, $\left(V,-K_{V}\right)$ makes it possible to describe the group of birational automorphisms of the variety $V$ of interest to us, and in our case $\alpha\left(V,-K_{V}\right)$ is clearly equal to 1 .

Our main result is stated in the following theorem.

Theorem. The singular double quadric $V$ described above is a birationally rigid variety, that is, for any test pair $(W, Y)$ and any birational map $\psi: V \rightarrow W$ there is a birational automorphism $\chi \in \operatorname{Bir}(V)$ such that

$$
\alpha\left(V,(\psi \circ \chi)^{-1}(Y)\right) \leqslant \alpha(W, Y) .
$$

Corollary. 1) $V$ is non-rational and is not birationally isomorphic to any fibring into conics or into Del Pezzo surfaces.

2) the group $\operatorname{Bir}(V)$ of birational automorphisms is included in the exact triple

$$
1 \longrightarrow * \tau_{B} \longrightarrow \operatorname{Bir}(V) \longrightarrow \operatorname{Aut}(V) \longrightarrow 1
$$

where $\operatorname{Aut}(V)$ is the group of biregular automorphisms of $V$ and $* \tau_{B}$ is the free product of the involutions $\tau_{B}$ over all lines $B \subset V$ of type $\mathrm{A}$ or $\mathrm{B}$.

\section{$\S 2$. Maximal singularities of a Double quadric With a Double point}

The origin of maximal singularities. As we have already observed (see $\S 1$ ), $\operatorname{Pic}(V)=\mathrm{Cl}(V)=\mathbb{Z}[H]$, where $H=\pi^{*}(L), \pi: V \rightarrow Q$ is our double cover, and $L$ is a hyperplane in $\mathbb{P}^{4}$.

Let $(W, Y)$ be a test pair, $\chi: V \rightarrow W$ a birational map, and $|D|=\chi^{-1}(|Y|)$ the proper inverse image of the linear system $|Y|$. Obviously, $|D| \subset|n H|$ for some $n \geqslant 1$, and $\alpha(V, D)=n$. 
We assume that $n>\alpha(W, Y)$. Then let $\varphi_{0}: V_{0} \rightarrow V$ be a blow-up of the singular point $P \in V$, and let $D^{0}=\varphi^{-1}(D)=\varphi^{*}(n H)-\nu_{0} E_{0}$, where $\nu_{0} \geqslant 0$ and $E_{0}$ is the exceptional divisor. We lift $\chi$ to $V_{0}$ and resolve the singularities of the birational $\operatorname{map} \chi_{0}=\chi \circ \varphi: V_{0} \rightarrow W:$

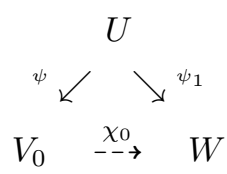

In this diagram $\psi$ and $\psi_{1}$ are birational morphisms. It is clear that there exist open subsets $W^{\prime} \subset W$ and $U^{\prime} \subset U$ such that $\left.\psi_{1}\right|_{U^{\prime}}: U^{\prime} \rightarrow W^{\prime}$ is an isomorphism, $U^{\prime}$ is smooth, and $\operatorname{codim}_{W}\left(W \backslash W^{\prime}\right) \geqslant 2$.

Since $n>\alpha(W, Y)$, we have that $\left|Y+n K_{W}\right|=\varnothing$, which implies the condition $\left|\psi_{1}^{-1}(Y)+n K_{W^{\prime}}\right|=\varnothing$. On the other hand, $\psi_{1}^{-1}(Y)=\psi^{-1}\left(D^{0}\right)$, and, moreover, there exist divisors $E_{1}, E_{2}, \ldots, E_{k} \subset U^{\prime}$ such that

$$
\begin{gathered}
K_{U^{\prime}}=\psi^{*} K_{V_{0}}+\sum_{i} \delta\left(E_{i}\right) E_{i}, \\
\psi^{-1}\left(D^{0}\right)=n \psi^{*} H-\nu_{0} \psi^{*} E_{0}-\sum_{i} \nu_{E_{i}}\left(D^{0}\right) E_{i} .
\end{gathered}
$$

Thus,

$$
\begin{aligned}
\varnothing & =\left|\psi_{1}^{-1}(Y)+n K_{U^{\prime}}\right| \\
& =\left|\left(n-\nu_{0}\right) \psi^{-1}\left(E_{0}\right)+\sum_{i=0}^{k}\left(n \delta\left(E_{i}\right)+\left(n-\nu_{0}\right) \nu_{E_{i}}\left(E_{0}\right)-\nu_{E_{i}}\left(D^{0}\right)\right) E_{i}\right| .
\end{aligned}
$$

We now need the following result.

Lemma. In our notation $\nu_{0} \leqslant n$.

Proof. We have the chain of morphisms $V_{0} \stackrel{\varphi_{0}}{\longrightarrow} V \stackrel{\pi}{\rightarrow} Q$. Let $S^{\prime}=Q \cap \overline{T_{P} Q}$, a quadratic cone in $\mathbb{P}^{3}$, and let $S=\pi^{-1}\left(S^{\prime}\right)$ and $S^{0}=\varphi^{-1}(S)$. We take a generator $l^{\prime} \subset S^{\prime}$ of the cone. Then either $l=\pi^{-1}\left(l^{\prime}\right)$ is a rational curve with a double point at $P$, or $l=l_{1} \cup l_{2}$ is a pair of lines on $V$ that intersect at $P$ (and at another point). Setting $l^{0}=\varphi^{-1}(l)$, or, respectively, $l_{i}^{0}=\varphi^{-1}\left(l_{i}\right)$, we get that

$$
D^{0} \circ l^{0}=2 n-2 \nu_{0}<0
$$

in the first case, and

$$
D^{0} \circ l_{i}^{0}=n-\nu_{0}<0
$$

in the second. This means that $S^{0} \subset \operatorname{Bas}\left|D^{0}\right|$, which contradicts the fact that $\left|D^{0}\right|$ is unfixed.

Thus, we conclude from (1) that for some $i$

$$
\nu_{E_{i}}\left(D^{0}\right)-\left(n-\nu_{0}\right) \nu_{E_{i}}\left(E_{0}\right)>n \delta\left(E_{i}\right) .
$$

Bearing in mind that $\nu_{E_{i}}\left(E_{0}\right) \geqslant 0$, we obtain the next result. 
Proposition. If $n>\alpha(W, Y)$, then $\nu_{E_{i}}\left(D^{0}\right)>n \delta\left(E_{i}\right)$ for some $i>0$, that is, the linear system $\left|D^{0}\right|$ has a maximal singularity in the sense of [2].

Remark. The number $n$ actually is the canonical threshold of the linear system $\left|D^{0}\right|$. Indeed, let $n_{1}>n$ be some number, and let $m=n_{1}-n>0$. Then obvious arguments give us that

$$
\left|D^{0}+n_{1} K_{V_{0}}\right|=\left|-m\left(\varphi_{0}^{*}(H)-E_{0}\right)+\left(n-\nu_{0}\right) E_{0}\right|=\varnothing .
$$

Thus, the proposition above is none other than the assertion that there exist maximal singularities in the linear system $\left|D^{0}\right|$.

Maximal singularities on the variety $V_{0}$. In the spirit of the more modern approach described in [5] we have at this point proved precisely the following: if $\chi: V \rightarrow W$ is a birational map, $|D|=\chi^{-1}|Y| \subset|n H|$, and $n>\alpha(W, Y)$, then there exists a triple $(\widetilde{V}, \varphi, T)$, where $T$ is a prime Weil divisor on $\widetilde{V}, T \not \subset \widetilde{V}_{\text {sing }}$, and $\varphi: \widetilde{V} \rightarrow V_{0}$ is a birational morphism, such that

$$
\nu_{T}\left|D^{0}\right|>n \delta(T)
$$

(here, as usual, $\nu_{T}\left|D^{0}\right| \stackrel{\text { def }}{=} \min _{C \in\left|D^{0}\right|} \nu_{T}(C)$ and $\delta(T) \stackrel{\text { def }}{=} \operatorname{ord}_{T}\left(\varphi^{*} \omega_{V_{0}} \otimes \omega_{\widetilde{V}}^{*}\right)$, and $\omega_{V_{0}}$ and $\omega_{\widetilde{V}}$ are the canonical sheaves on $V_{0}$ and $\widetilde{V}$ ).

Accordingly, suppose that the triple $(\widetilde{V}, \varphi, T)$ gives a maximal singularity of the linear system $\left|D^{0}\right| \subset\left|n \varphi_{0}^{*}(H)-\nu_{0} E_{0}\right|$, with $n$ the canonical threshold, and let $B_{0}=\varphi(T)\left(B_{0}\right.$ is none other than the centre of the valuation determined by the divisor $T)$. Two essentially different cases are possible.

Case 1. $\operatorname{supp} B_{0} \not \subset E_{0}$. Then, as is not hard to see, we can lower everything to $V$ and employ the arguments of [1] almost word for word. Thus, for $B=\varphi_{0}\left(B_{0}\right)$ we have only one possibility: $\operatorname{dim} B=1$, and here $B$ is necessarily a line of type A or B.

Case 2. supp $B_{0} \subset E_{0}$. This is the case of so-called infinitely near maximal singularities (of the linear system $|D|$ on $V$ ). It is proved below that there are no such singularities in our case.

\section{§3. Elimination of INFINitely NEAR Singularities}

Proposition. In the preceding notation let $\left|D^{0}\right| \subset\left|n \varphi_{0}^{*}(H)-\nu_{0} E_{0}\right|$ be an unfixed linear system, and let $\nu_{0} \leqslant n$. There do not exist triples $(\widetilde{V}, \varphi, T)$ (see $\left.\S 2\right)$ such that the inequality (2) holds if $\operatorname{supp} \varphi(T) \subset E_{0}$.

Proof. Suppose the contrary. Then there is a (finite) chain of birational morphisms (see [5])

(3) $\tilde{V}=V_{N} \stackrel{\varphi_{N, N-1}}{\longrightarrow} V_{N-1} \stackrel{\varphi_{N-1, N-2}}{\longrightarrow} \ldots \stackrel{\varphi_{i+1, i}}{\longrightarrow} V_{i} \stackrel{\varphi_{i, i-1}}{\longrightarrow} \ldots \stackrel{\varphi_{1,0}}{\longrightarrow} V_{0}$ 
such that $\varphi(T)=\varphi_{N, N-1} \circ \cdots \circ \varphi_{1,0}(T) \subset E_{0}$, each morphism $\varphi_{k, k-1}$ is a blow-up with centre $B_{k-1}$ at a point or irreducible (possibly singular) curve on $V_{k-1}$, and $\nu_{T}\left|D^{0}\right|>n \delta(T)$. Let $E_{i}=\varphi_{i, i-1}^{-1}\left(B_{i-1}\right) \subset V_{i}$ and $E_{N}=T$. We can assume that $B_{i}$ is a point for $i<L$ and a curve for $i \geqslant L$, where $0 \leqslant L \leqslant N$; furthermore, we can assume that $B_{i} \subset E_{i}$ for any $i$.

Let $\nu_{i}=$ mult $_{B_{i-1}}|D|^{i}$, where $|D|^{i}$ is the proper inverse image of the linear system $\left|D^{0}\right|$ on $V_{i}$. It is clear that $\nu_{1} \geqslant \nu_{2} \geqslant \cdots \geqslant \nu_{N}$. Moreover, it is easy to see that $\nu_{1} \leqslant 2 \nu_{0}$.

Let $\Gamma$ be the directed graph of the singularity: vertices $i$ and $j$ are joined by an arrow (from $i$ to $j$ ) if $i>j$ and $B_{i-1} \subset E_{j}^{i-1}$. We denote by $p(i, j)$ the number of different paths from a vertex $i$ to a vertex $j$ when $i>j ; p(i, i) \stackrel{\text { def }}{=} 1$.

As is well known, the numbers $\delta_{i}=\operatorname{codim} B_{i}-1$ and $\nu_{i}$ are connected by the Noether-Fano-Iskovskikh inequality ([1], [2], [5]):

$$
\sum_{i=1}^{N} p(N, i) \nu_{i}>\sum_{i=1}^{N} p(N, i) \delta_{i-1} .
$$

We remark that the case $\operatorname{dim} B_{0}=0, N=1$, that is, the maximal singularity is at a point and is realized by the very first blow-up, is impossible, because then $2 n<\nu_{1} \leqslant 2 \nu_{0}$, so that $\nu_{0}>n$, which contradicts the lemma in $\S 2$.

Suppose now that $\operatorname{dim} B_{0}=1$. Then it follows from (4) that $\nu_{1}>n$. The surface $E_{0}$ is isomorphic to a quadric in $\mathbb{P}^{3}$, so that $\operatorname{Pic}\left(E_{0}\right) \simeq \mathbb{Z} \oplus \mathbb{Z}$. The linear system $\left|D^{0}\right|$ restricted to $E_{0}$ has type $\left(\nu_{0}, \nu_{0}\right), \nu_{0} \leqslant n$; on the other hand, $\left.\operatorname{ord}_{B_{0}} D^{0}\right|_{E_{0}} \geqslant \nu_{1}>n$, which is not possible.

The remaining case, that is, when $\operatorname{dim} B_{0}=0$ and $N>1$, is very difficult; its impossibility is proved in the next section.

\section{$\S 4$. The Difficult CASE}

The 'difficulty' of this case is explained by the following circumstances. Suppose, for example, that we need to eliminate a maximal singularity of some linear system $|M|$ over a point $A$. To do this, from the point of view of the approach described in [5], we must do two things: first, show with the help of the Noether-FanoIskovskikh inequalities that two general elements $M_{1}, M_{2} \in|M|$ cut out a curve $C=M_{1} \circ M_{2}$ having high multiplicity at $A$; second, find a surface $S$ (a so-called test surface) that is smooth at $A$, does not contain components of $C$, and is such that $C \circ S<$ mult $_{P} C$.

In our case it is convenient to take as test surfaces the elements of the class $\left|\varphi_{1,0}^{*}(H)-E_{0}\right|$, but the following lemma indicates the source of the troubles.

Lemma. Let $Z$ be an irreducible reduced curve and let $A \in Z \cap E_{0}$ be some point. The following conditions are equivalent:

(i) $Z \subset S$ for any surface $S \in\left|\varphi_{1,0}^{*}(H)-E_{0}\right|$ passing through $A$;

(ii) $\pi \circ \varphi_{1,0}(Z)$ is a line on the quadric $Q$ and passing through $P$.

Proof. It suffices to carry out simple local computations in a neighbourhood of $P$.

On the other hand, if the curve $Z$ satisfies the condition ii) of the preceding lemma and the linear system $D^{0}$ has a maximal singularity over the point $A$, then it is always true that $Z \subset \operatorname{Bas}\left|D^{0}\right|$. Indeed, if $\varphi_{1,0}(Z)$ is a 'line', that is, $H \circ \varphi_{1,0}(Z)=1$, 
then $D^{0} \circ Z=n-\nu_{0} \leqslant n<\nu_{1}$, which is possible only if $Z \subset D^{0}$. But if $\varphi_{1,0}(Z)$ is a 'conic', that is, $H \circ \varphi_{1,0}(Z)=2$, and in addition $Z \not \subset D^{0}$, then we get a contradiction: $D^{0} \circ Z=2 n-2 \nu_{0} \geqslant \nu_{1}>n$, whence $\nu_{0}<n / 2$ (recall that $\left.2 \nu_{0} \geqslant \nu_{1}>n\right)$. Thus, it is not possible to use the test surface method directly in these cases.

We remark that the points on $E_{0}$ through which 'lines' or 'conics' pass, lie on some curve $C_{F_{Q}} \subset E_{0}$ that is a projectivization of the tangent cone to the branch divisor (so that $C_{F_{Q}}$ is a conic on $E_{0} \cong \mathbb{P}^{1} \times \mathbb{P}^{1}$ ). The next assertion is almost obvious.

Proposition. A linear system cannot contain maximal singularities over a point $B_{0} \in E_{0} \backslash C_{F_{Q}}$.

Proof. The proof is by contradiction. Let $m=\operatorname{mult}_{B_{0}}\left(D_{1}^{0} \circ D_{2}^{0}\right)$, where $D_{1}^{0}, D_{2}^{0}$ in $\left|D^{0}\right|$ are sufficiently general. The inequality $m>4 n^{2}$ can be deduced from (4) (see [1], [2], [5]). If $S \in\left|\varphi_{1,0}^{*}(H)-E_{0}\right|$ is a smooth surface (this is a surface of type K3), $B_{0} \in S$, and $S$ is in general position with $D_{1}^{0} \circ D_{2}^{0}$ (such a surface exists according to the preceding lemma), then we get a contradiction:

$$
m \leqslant D_{1}^{0} \circ D_{2}^{0} \circ S=n^{2}(H)^{3}-\nu_{0}^{2}\left(E_{0}\right)^{3}=4 n^{2}-2 \nu_{0}^{2}<4 n^{2} .
$$

Suppose now that the linear system $\left|D^{0}\right|$ has a maximal singularity over a point $B_{0} \in E_{0}$ and let $l^{0} \ni B_{0}$ be a 'conic' or 'line' on $V_{0}$ (in the last case there exists a 'line' $l^{* 0}$ that is conjugate to it with respect to the double cover automorphism). Let $\varepsilon=\operatorname{mult}_{l^{0}}\left(D_{1}^{0} \circ D_{2}^{0}\right)$ (respectively, $\varepsilon^{*}=$ mult $\left._{l^{* 0}}\left(D_{1}^{0} \circ D_{2}^{0}\right)\right)$. We would like to get lower estimates for the quantity $\varepsilon$ or for a combination of it with other 'harmful' parameters. But before doing this we shall see what upper estimates we can count on.

Proposition. (i) Let $l^{0}$ be a 'conic'. Then

$$
\varepsilon \leqslant 2 n^{2} \text {. }
$$

(ii) Let $l^{0}$ be a 'line', and let $\mu=\operatorname{mult}_{l^{0}}\left|D^{0}\right|$. Then

$$
\varepsilon-\varepsilon^{*} \leqslant 4 n^{2}-\nu_{0}^{2}-2 \mu n-\left(\nu_{0}-\mu\right)^{2}-\left(\nu_{1}-\mu\right)^{2} .
$$

Proof. (i) Suppose that $D_{1}, D_{2} \in|D| \subset|n H|$ and $S \in|H|$ are in general position (we are considering everything on $V$ ). Then

$$
4 n^{2}=D_{1} \circ D_{2} \circ S \geqslant \varepsilon l \circ S=2 \varepsilon .
$$

(ii) Suppose that $\left|S^{0}\right| \in\left|H^{*}-E_{0}\right|$ is a general element passing through the point $B_{0}$; it can be assumed that $S^{0} \cap \operatorname{Bas}\left|D^{0}\right|=l^{0} \cup l^{* 0}$ (here and below the raised symbol $*$ in the notation for a divisor means the complete inverse image on the corresponding variety, in this case on $V_{0}$ ). A natural step is to blow up $l^{0}$. Let $\psi: V^{\prime} \rightarrow V_{0}$ be such a blow-up, and let $E^{\prime}$ be its exceptional divisor. 
Lemma. $E^{\prime} \cong \mathbb{P}^{1} \times \mathbb{P}^{1}$.

Proof. It is not hard to see that the normal sheaf $\boldsymbol{N}_{l^{0} \mid V_{0}}$ can be represented as the extension

$$
0 \longrightarrow \boldsymbol{O}_{l^{0}}(-2) \longrightarrow \boldsymbol{N}_{l^{0} \mid V_{0}} \longrightarrow \boldsymbol{O}_{l^{0}} \longrightarrow 0
$$

so that the exceptional divisor $E^{\prime}$ is isomorphic either to the surface $F_{2}$ or to the quadric $F_{0}$.

We assume that $E^{\prime} \cong F_{2}$ and we let $s$ and $f$ be the classes of the exceptional section and of the fibre of the surface $E^{\prime}$. We consider a maximally general element $T \in\left|H^{*}-E_{0}\right|$ containing the curve $l^{0}$ (this is a smooth surface of type K3). Let $\left.T^{\prime}\right|_{E}=s+\alpha f$, where $T^{\prime}$ is the proper inverse image. For two such elements $T_{1}$ and $T_{2}$ it is obvious that

$$
2=T_{1}^{\prime} \circ T_{2}^{\prime} \circ E^{\prime}=(s+\alpha f)^{2}=\alpha,
$$

so that $\left.T^{\prime}\right|_{E}=s+2 f$.

Further, local arguments give us easily that there is a sheaf $|M| \subset\left|H^{*}-E_{0}\right|$ on $V_{0}$ such that:

1) $l^{0}, l^{* 0} \subset \operatorname{Bas}|M|$

2) a general element of $|M|$ has a double point at the unique point of intersection of $l^{0}$ and $l^{* 0}$ (this means that $\pi \circ \varphi_{0}(M)$ is tangent to $F_{Q}$ at the point where $\pi \circ \varphi_{0}\left(l^{0}\right)$ is tangent to $\left.F_{Q}\right)$;

3) two general elements of $|M|$ do not have tangencies along $l^{0}$.

Suppose that $\left.M^{\prime}\right|_{E}=s+\alpha f$ for the proper inverse image of this sheaf. It is not hard to see that $2=M^{\prime} \circ T^{\prime} \circ E^{\prime}=\alpha$. On the other hand, $\left|M^{\prime}\right|$ has the fibre over the singular point of $M$ as a base curve and the system $|s+f|$ on $F_{2}$ does not contain irreducible curves. Thus, $s \subset$ Bas $\left|M^{\prime}\right|$, that is, general elements of $|M|$ are tangent along $l^{0}$. This contradiction proves the lemma.

It is not hard to see that the intersection of the cycles is equal to

$$
\begin{aligned}
D_{1}^{\prime} \circ D_{2}^{\prime} \circ S^{\prime} & =\left(n H^{*}-\nu_{0} E_{0}^{*}-\mu E^{\prime}\right)^{2}\left(H^{*}-E_{0}^{*}-E^{\prime}\right) \\
& =4 n^{2}-2 \nu_{0}^{2}-2 \mu n+2 \mu \nu_{0}-2 \mu^{2},
\end{aligned}
$$

where $\left|S^{\prime}\right|$ is the proper inverse image of the system $\left|S^{0}\right|$.

On the other hand, let $\bar{f}$ be the fibre of $E^{\prime}$ over the point $B_{0}$. It is easy to see that $\bar{f} \subset \operatorname{Bas}\left|D^{\prime}\right|$ and $m_{\bar{f}}=\operatorname{ord}_{\bar{f}}\left(D_{1}^{\prime} \circ D_{2}^{\prime}\right) \geqslant\left(\nu_{1}-\mu\right)^{2}$ for general elements $D_{1}^{\prime}, D_{2}^{\prime}$ of the proper inverse image $\left|D^{\prime}\right|$ of the system $\left|D^{0}\right|$. Moreover, for the blow-up $\psi$ the linear system $\left|D^{\prime}\right|$ acquires another base curve $Z^{\prime}, \operatorname{supp}\left(Z^{\prime}\right) \subset E^{\prime}$, which has degree $d$ with respect to the fibres of $E^{\prime}$, and $\mu^{2}+d=\varepsilon$ (Lemma 6.1(ii) in [5]). Thus,

$$
D_{1}^{\prime} \circ D_{2}^{\prime}=m_{\bar{f}} \bar{f}+Z^{\prime}+\varepsilon^{*} l^{* \prime}+Z,
$$

where $l^{* \prime}$ is the proper inverse image of $l^{* 0}$ and $Z$ is some curve whose support does not contain $\bar{f}, l^{* \prime}$, or components of the curve $Z^{\prime}$.

Since $S^{\prime} \cap \operatorname{Bas}\left|D^{\prime}\right|=l^{*^{\prime}},\left.Z^{\prime} \circ S^{\prime}\right|_{E^{\prime}}=Z^{\prime} \circ(s+f) \geqslant d, S^{\prime} \circ \bar{f}=1$, and $S^{\prime} \circ l^{*^{\prime}}=-1$, it follows that

$$
D_{1}^{\prime} \circ D_{2}^{\prime} \circ S^{\prime} \geqslant\left(\nu_{1}-\mu\right)^{2}+d-\varepsilon^{*} .
$$

Comparison of the expressions obtained yields (ii). 
Our next problem, as follows from the proposition just proved, is to get the strongest possible lower estimates for $\varepsilon$ or, respectively, for $\varepsilon-\varepsilon^{*}$.

As before, it is possible to choose a realization of our maximal singularity (the sequence (3)) in which each subsequent centre of a blow-up dominates the preceding one, and we can let $B_{0}, \ldots, B_{L-1}$ be points and $B_{L}, \ldots, B_{N-1}$ (irreducible) curves.

The nature of our subsequent actions depends essentially on the moment at which the proper inverse image of the curve $l^{0}$ 'jumps from' the next centre of a blow-up. Let $k=\max \left(0 \ldots L-1: B_{k} \in l^{k}, B_{k+1} \notin l^{k+1}\right)$ (as usual, superscripts denote the proper inverse image in the corresponding step of the chain of blow-ups).

Case $k=0$. In this case the first blow-up takes away the curve of interest to us from the next centre $B_{1}$. We introduce the notation that has now become standard for the method of maximal singularities. Namely, for general elements $D_{1}^{0}, D_{2}^{0} \in\left|D^{0}\right|$ let

$$
D_{1}^{0} \circ D_{2}^{0}=Z_{0}+\widetilde{Z}_{0}+\varepsilon l^{0}
$$

where $Z_{0}$ is a curve with $l^{0} \not \subset \operatorname{supp} Z_{0}$ and no component of $Z_{0}$ lies in $E_{0}$; further, $\widetilde{Z}_{0} \subset E_{0}$ is a base curve (possibly empty) of the system $\left|D^{0}\right|, \widetilde{d}$ is its degree with respect to the class $(1,1)$ on $E_{0}$, and $\widetilde{m}_{0, i}=$ mult $_{B_{i-1}} \widetilde{Z}_{0}^{i-1}$ for $i=1, \ldots, L$ $\left(\widetilde{d}=\widetilde{m}_{0, i}=0\right.$ if $\left.\widetilde{Z}_{0}=\varnothing\right)$; suppose also that $Z_{i} \subset E_{i}, 1 \leqslant i \leqslant L$, are curves such that $D_{1}^{i} \circ D_{2}^{i}=\left(D_{1}^{i-1} \circ D_{2}^{i-1}\right)^{i}+Z_{i}$, and let $d_{i}=\operatorname{deg} Z_{i}$ and $m_{i, j}=\operatorname{mult}_{B_{j-1}} Z_{i}^{j-1}$ for $L \geqslant j>i \geqslant 0$.

The quantities introduced are connected by the following relations [5]:

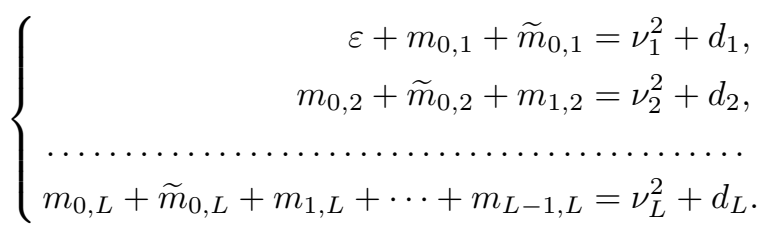

We introduce the notation $r_{i}=p(N, i)$ (the numbers $p(i, j)$ were defined in $\S 3$ ). Let us multiply the rows of the system (8) by $r_{1}, r_{2}, \ldots, r_{L}$, respectively, and add them. We get in a standard way (Theorem 7.1 in [5]) that

$$
r_{1} \varepsilon+\sum_{i=1}^{L} r_{i}\left(m_{0, i}+\widetilde{m}_{0, i}\right) \geqslant \sum_{i=1}^{N} r_{i} \nu_{i}^{2} .
$$

In place of (4) we shall need the refined inequality (it is derived in exactly the same way as (4), except that $E_{0}$ is also taken into account)

$$
r_{0} \nu_{0}+\sum_{i=1}^{N} r_{i} \nu_{i}>2 n \Sigma_{0}+n \Sigma_{1}+r_{0} n
$$

where $\Sigma_{0}=\sum_{i=1}^{L} r_{i}$ and $\Sigma_{1}=\sum_{i=L+1}^{N} r_{i}$. 
Since $\nu_{0} \leqslant n$, we can decrease $r_{0}$ somewhat by setting $r_{0}=r_{1}$; this just makes the inequality (10) stronger.

Then since $2 \nu_{0} \geqslant \nu_{1} \geqslant \cdots \geqslant \nu_{N}$, we obtain in the usual way the quadratic inequality

$$
2 r_{0} \nu_{0}+\sum_{i=1}^{N} r_{i} \nu_{i}^{2}>\frac{\left(2 \Sigma_{0}+\Sigma_{1}+r_{0}\right)^{2} n^{2}}{\Sigma_{0}+\Sigma_{1}+\frac{1}{2} r_{0}}
$$

from which it follows that

$$
\sum_{i=1}^{N} r_{i} \nu_{i}^{2}>4 n^{2} \Sigma_{0}+2 r_{0} n^{2}-2 r_{0} \nu_{0}^{2}+\frac{\Sigma_{1}^{2} n^{2}}{\Sigma_{0}+\Sigma_{1}+\frac{1}{2} r_{0}} .
$$

The inequalities (9) and (11) together yield

$$
r_{1} \varepsilon+\sum_{i=1}^{L} r_{i}\left(m_{0, i}+\widetilde{m}_{0, i}\right) \geqslant 4 n^{2} \Sigma_{0}+2 r_{0} n^{2}-2 r_{0} \nu_{0}^{2}+\frac{\Sigma_{1}^{2} n^{2}}{\Sigma_{0}+\Sigma_{1}+\frac{1}{2} r_{0}} .
$$

As earlier, let $S^{0}$ be a general element of the system $\left|H^{*}-E_{0}\right|$. It is clear that

$$
m_{0, i}+\widetilde{m}_{0, i} \leqslant m_{0,1}+\widetilde{m}_{0,1} \leqslant D_{1}^{0} \circ D_{2}^{0} \circ S^{0} \leqslant 4 n^{2}-2 \nu_{0}^{2} .
$$

Then after division by $r_{1}=r_{0}$ we get from the inequality (12) that

$$
\varepsilon>2 n^{2},
$$

which takes care of us if $l^{0}$ is a 'conic'.

In the case when $l^{0}$ is a 'line' we leave the term $m_{0,1}+\widetilde{m}_{0,1}$ in (12) and also divide by $r_{1}$ :

$$
\varepsilon+m_{0,1}+\widetilde{m}_{0,1}>6 n^{2}-2 \nu_{0}^{2}+\frac{\Sigma_{1}^{2} n^{2}}{\left(\Sigma_{0}+\Sigma_{1}+\frac{1}{2} r_{0}\right) r_{1}} .
$$

As in the proof of the inequality (6), let $\psi: V^{\prime} \rightarrow V_{0}$ be a blow-up of the 'line' $l^{0}$. It is easy to compute that

$$
D_{1}^{\prime} \circ D_{2}^{\prime} \circ E^{\prime}=2 \mu^{2}+2 \mu n-2 \mu \nu_{0} .
$$

We carry out a more refined computation of the curves:

$$
D_{1}^{\prime} \circ D_{2}^{\prime}=\varepsilon^{*} l^{* \prime}+Z^{\prime}+Z_{0}^{\prime}+\widetilde{Z}_{0}^{\prime}+C+\text { (some curves), }
$$

where the curve $C$ is a sum of fibres of $E^{\prime}$ with multiplicities. Denoting the zero section and the fibre of $E^{\prime}$ as before by $s$ and $f$, we can assume that

$$
Z^{\prime}+C=d s+\left(\nu_{1}-\mu\right)^{2} f+M f
$$

where $M \geqslant 0$ is some number. Next, it is clear that $\widetilde{Z}_{0}^{\prime} \circ E^{\prime}=\widetilde{m}_{0,1}$ and that $Z_{0}^{\prime} \circ E^{\prime}=m_{0,1}$, therefore,

$$
2 \mu^{2}+2 \mu n-2 \mu \nu_{0} \geqslant \varepsilon^{*}-d-\left(\nu_{1}-\mu\right)^{2}-M+\widetilde{m}_{0,1}+m_{0,1} .
$$


On the other hand,

$$
4 n^{2}-2 \nu_{0}^{2}-2 \mu^{2}-2 \mu n+2 \mu \nu_{0}=D_{1}^{\prime} \circ D_{2}^{\prime} \circ S^{\prime} \geqslant\left(\nu_{1}-\mu\right)^{2}+d+\widetilde{d}-\widetilde{m}_{0,1}+M,
$$

where $S^{\prime}$ is a general element of $\left|H^{*}-E_{0}^{*}-E^{\prime}\right|$. The last two inequalities yield

$$
4 n^{2}-2 \nu_{0}^{2} \geqslant \varepsilon^{*}+\widetilde{d}+m_{0,1}
$$

Substituting this in (13) and taking into account that $\widetilde{m}_{0,1} \leqslant \widetilde{d}$ and $\Sigma_{0} \geqslant r_{1}$, we get the desired estimate

$$
\varepsilon-\varepsilon^{*}>2 n^{2}+\frac{\Sigma_{1}^{2} n^{2}}{\left(\Sigma_{0}+\Sigma_{1}+\frac{1}{2} r_{0}\right) \Sigma_{0}} .
$$

Case $k>0$. It is a simple matter to see that the preceding inequalities were valid because $l^{1}$ jumped from the centre of the blow-up. We shall try to do something similar also in the case $k>0$, that is, after making some refinements, we begin everything as it were from the last step where our curve still 'sits' on the centre of the blow-up.

We make two preliminary observations about the coefficients $r_{i}$ in the NoetherFano-Iskovskikh inequality (10). First, starting from the definition of these numbers, we can write that

$$
r_{k}=\sum_{j \rightarrow k} r_{j}=\sum_{\substack{j \rightarrow k \\ j \leqslant L}} r_{j}+\sum_{\substack{j \rightarrow k \\ j>L}} r_{j} .
$$

The inequality (10) permits us easily to decrease $r_{k}$; let

$$
r_{k}=\sum_{\substack{j \rightarrow k \\ j \leqslant L}} r_{j}
$$

Second, we can let $r_{0}=r_{1}=\cdots=r_{k}$ (incidentally, it is not hard to see that this holds automatically for our choice of realization of the maximal singularity).

Using the notation for the case $k=0$, we write the two systems of equalities:

$$
\left\{\begin{array}{r}
\varepsilon+m_{0,1}+\widetilde{m}_{0,1}=\nu_{1}^{2}+d_{1}, \\
\varepsilon+m_{0,2}+m_{1,2}=\nu_{2}^{2}+d_{2}, \\
\ldots \ldots \ldots \ldots \ldots \ldots \ldots \ldots \ldots \\
\varepsilon+m_{0, k}+m_{k-1, k}=\nu_{k}^{2}+d_{k},
\end{array}\right.
$$


and

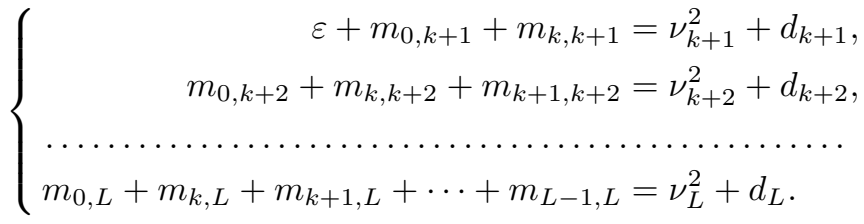

We note that $\widetilde{m}_{0, i}=0$ for $i>1$, since $k>0$.

Next, we multiply each row of the system (14) by $r_{k}$, while in the system (15) we multiply the first row by $r_{k+1}$, the second by $r_{k+2}$, and so on, after which we add the left-hand and right-hand sides of both systems. The standard trick with annihilation of the quantities $d_{i}$ and $m_{i, j}$ still works, despite the decrease of the coefficient $r_{k}$, and, using the quadratic inequality (11) and taking into account that $\Sigma_{0}=k r_{k}+\sum_{i=k+1}^{L} r_{i}$, we get that

$$
\begin{aligned}
\varepsilon\left(k+\frac{r_{k+1}}{r_{k}}\right) & +\widetilde{m}_{0,1}+\sum_{i=1}^{k} m_{0, i}+\sum_{i=k+1}^{L} \frac{r_{i}}{r_{k}} m_{0, i} \\
& >4 n^{2}\left(k+\sum_{i=k+1}^{L} \frac{r_{i}}{r_{k}}\right)+2 n^{2}-2 \nu_{0}^{2}+\frac{\Sigma_{1}^{2} n^{2}}{\left(\Sigma_{0}+\Sigma_{1}+\frac{1}{2} r_{0}\right) r_{k}} .
\end{aligned}
$$

We now observe that

$$
\sum_{i=k+1}^{L} \frac{r_{i}}{r_{k}}\left(4 n^{2}-m_{0, i}\right) \geqslant\left(4 n^{2}-m_{0,1}\right) \sum_{\substack{j \rightarrow k \\ j \leqslant L}} \frac{r_{j}}{r_{k}}=4 n^{2}-m_{0,1}
$$

and for a general $S^{0} \in\left|H^{*}-E_{0}\right|$

$$
4 n^{2}-2 \nu_{0}^{2}=D_{1}^{0} \circ D_{2}^{0} \circ S^{0} \geqslant m_{0,1}+\widetilde{m}_{0,1} ;
$$

we get (using $r_{k} \geqslant r_{k+1}$ ) that

$$
\varepsilon(k+1)+\sum_{i=1}^{k} m_{0, i}>4 n^{2} k+2 n^{2}+\frac{\Sigma_{1}^{2} n^{2}}{\left(\Sigma_{0}+\Sigma_{1}+\frac{1}{2} r_{0}\right) r_{k}} .
$$

Lemma. $\sum_{i=1}^{k} m_{0, i} \leqslant 4 n^{2}-\varepsilon-\varepsilon^{*}$ (if $l^{0}$ is a 'conic', then in place of $\varepsilon^{*}$ substitute another $\varepsilon)$.

Proof. Let $S^{0} \in\left|H^{*}-E_{0}\right|$ and let $B_{0} \in S^{0}$ be a general element. It is easy to compute that in the $k$ th step

$$
D_{1}^{k} \circ D_{2}^{k} \circ S^{k}=4 n^{2}-2 \nu_{0}^{2}-\sum_{i=1}^{k} \nu_{i}^{2} .
$$

On the other hand,

$$
D_{1}^{k} \circ D_{2}^{k}=Z_{0}^{k}+\widetilde{Z}_{0}^{k}+\varepsilon l^{k}+\varepsilon^{*} l^{* k}+Z_{1}^{k}+\cdots+Z_{k}
$$

(if $l^{0}$ is a 'conic', then the corresponding term must be thrown out, of course). 
It is clear that $S^{k}=H^{*}-\sum_{i=0}^{k} E_{i}^{*}$, from which $l^{k} \circ S^{k}=-k, l^{* k} \circ S^{k}=0$, $Z_{0}^{k} \circ S^{0} \geqslant m_{0, k} \geqslant 0$, and $\widetilde{Z}_{0}^{k}=\widetilde{d}-\widetilde{m}_{0,1}\left(\right.$ recall that $\left.\widetilde{d}=\operatorname{deg}_{E_{0}} \widetilde{Z}_{0}\right)$.

Further, $Z_{i}^{i+1} \circ S^{i+1}=d_{i}-m_{i, i+1}$ for $1 \leqslant i<k$ and hence

$$
Z_{i}^{k} \circ S^{k}=Z_{i}^{i+1} \circ S^{i+1}-Z_{i}^{k} \circ\left(\sum_{j=i+2}^{k} E_{j}^{*}\right)=d_{i}-m_{i, i+1} .
$$

Finally, since $Z_{k} \circ S^{k}=d_{k}$, we get that

$$
4 n^{2}-2 \nu_{0}^{2}-\sum_{i=1}^{k} \nu_{i}^{2} \geqslant-k \varepsilon+\widetilde{d}-\widetilde{m}_{0,1}+\sum_{i=1}^{k-1}\left(d_{i}-m_{i, i+1}\right)+d_{k} .
$$

Adding both sides of the system (14) yields

$$
k \varepsilon+\widetilde{m}_{0,1}+\sum_{i=1}^{k} m_{0, i}=\sum_{i=1}^{k} \nu_{i}^{2}+\sum_{i=1}^{k-1}\left(d_{i}-m_{i, i+1}\right)+d_{k} .
$$

Comparing these two expressions, we see that

$$
4 n^{2}-2 \nu_{0}^{2} \geqslant \sum_{i=1}^{k} m_{0, i}+\widetilde{d}
$$

The assertion of the lemma is now obtained from the estimate for $\widetilde{d}$ :

$$
\begin{aligned}
& 2 \nu_{0}^{2}=D_{1}^{0} \circ D_{2}^{0} \circ E_{0} \geqslant \varepsilon+\varepsilon^{*}-\widetilde{d} \text { if } l^{0} \text { is a 'line'; } \\
& 2 \nu_{0}^{2}=D_{1}^{0} \circ D_{2}^{0} \circ E_{0} \geqslant 2 \varepsilon-\widetilde{d} \quad \text { if } l^{0} \text { is a 'conic'. }
\end{aligned}
$$

Everything is now ready for the needed estimates. Accordingly, let $l^{0}$ be a conic; the inequality (16) together with the statement of the lemma gives us that

$$
(k-1) \varepsilon>4 n^{2} k-2 n^{2} .
$$

The assumption $k=1$ leads immediately to a contradiction: $0>2 n^{2}$. But if $k>1$, then it is not hard to see that $\varepsilon>2 n^{2}$.

Suppose now that $l^{0}$ is a line. Then

$$
k \varepsilon>4 n^{2} k-2 n^{2}+\varepsilon^{*}+\frac{\Sigma_{1}^{2} n^{2}}{\left(\Sigma_{0}+\Sigma_{1}+\frac{1}{2} r_{0}\right) r_{k}} .
$$

Since $k r_{k} \leqslant \Sigma_{0}$, it follows that

$$
\varepsilon>4 n^{2}-\frac{2 n^{2}}{k}+\frac{\varepsilon^{*}}{k}+\frac{\Sigma_{1}^{2} n^{2}}{\left(\Sigma_{0}+\Sigma_{1}+\frac{1}{2} r_{0}\right) \Sigma_{0}} .
$$

For $k=1$ we get at once the needed estimate $\varepsilon-\varepsilon^{*}>2 n^{2}+\frac{\Sigma_{1}^{2} n^{2}}{\left(\Sigma_{0}+\Sigma_{1}+\frac{1}{2} r_{0}\right) \Sigma_{0}}$.

For $k>1$ we must also take into account the inequality $\varepsilon+\varepsilon^{*} \leqslant 4 n^{2}$.

Thus, we have proved the following result. 


\section{Proposition.}

(i) $\varepsilon>2 n^{2}$ if $l^{0}$ is a 'conic';

(ii) $\varepsilon-\varepsilon^{*}>2 n^{2}+\frac{\Sigma_{1}^{2} n^{2}}{\left(\Sigma_{0}+\Sigma_{1}+\frac{1}{2} r_{0}\right) \Sigma_{0}}$ if $l^{0}$ is a 'line'.

Corollary 1. The linear system $\left|D^{0}\right|$ cannot have maximal singularities over a point $B_{0}$ through which a 'conic' passes.

Proof. The case $k=1$ is impossible, as we have just seen; for other values of $k$ we get a contradiction of the inequality (5).

Corollary 2. The linear system $\left|D^{0}\right|$ cannot have maximal singularities over a point $B_{0}$ through which a 'line' passes.

Proof. We use a device described in [3]. The inequality (10) implies that

$$
\Sigma_{1}>\frac{2 n-\nu_{1}}{\nu_{1}-n} \Sigma_{0}+\frac{n-\nu_{0}}{\nu_{1}-n} r_{0} .
$$

Next, we let $\nu_{0}+\nu_{1}=3 \theta$ with $n / 2<\theta \leqslant n$, and we consider the auxiliary function $\Lambda(t)=4 n^{2}-2 n \mu-t^{2}-(t-\mu)^{2}-(2 t-\mu)^{2}$.

We remark that $\Lambda(t)$ is decreasing for $t>n / 2$ and that $\varepsilon-\varepsilon^{*} \leqslant \Lambda(\theta)$. Moreover,

$$
\Sigma_{1}>\frac{2 n-2 \theta}{2 \theta-n} \Sigma_{0}+\frac{n-\theta}{2 \theta-n} r_{0} .
$$

Lemma. $\theta>\frac{2}{3} n$.

Proof. Suppose not. Then $\Sigma_{1}>2 \Sigma_{0}+r_{0}$ by the preceding inequality for $\Sigma_{1}$, and hence

$$
\varepsilon-\varepsilon^{*}>2 n^{2}+\frac{\Sigma_{1}^{2} n^{2}}{\left(\Sigma_{0}+\Sigma_{1}+\frac{1}{2} r_{0}\right) \Sigma_{0}} \geqslant 2 n^{2}+\frac{2}{3} n^{2} \frac{\Sigma_{1}}{\Sigma_{0}}>\frac{10}{3} n^{2} .
$$

On the other hand, $\varepsilon-\varepsilon^{*}<\Lambda\left(\frac{n}{2}\right) \leqslant \frac{21}{8} n^{2}$, which is a contradiction.

Thus, $\theta>\frac{2}{3} n$. But then

$$
2 n^{2}<\varepsilon-\varepsilon^{*} \leqslant \Lambda\left(\frac{2}{3} n\right) \leqslant \frac{33}{18} n^{2}<2 n^{2} .
$$

The corollary is proved.

\section{$\S 5$. UnTwisting AUTOMORPHISMS}

By now we have established the following: if $\chi: V \rightarrow W$ is a birational map onto a test pair $(W, Y),|D|=\chi^{-1}|Y| \subset|n H|$, and $n>\alpha(W, Y)$, then there is a line $B \subset V$ of type A or B at which the linear system $|D|$ has a maximal singularity.

On the other hand, a birational automorphism $\tau_{B}$ associated in a natural way with the line $B$ was constructed in $\S 1$. If we now show that $\left(\chi \circ \tau_{B}\right)^{-1}|Y| \subset|m H|$ and $m<n$, then this means that for any such birational automorphism there exist lines $B_{1}, \ldots B_{k}$, not necessarily all distinct, such that $\left(\chi \circ \tau_{B_{1}} \circ \cdots \circ \tau_{B_{k}}\right)^{-1}|Y|$ is contained in $|\alpha(W, Y) H|$.

The next proposition shows that this is indeed the case. 
Proposition. Let $|D| \subset|n H|$ be an unfixed linear system with $n>1$ having a maximal singularity at a line $B \subset V$ of type $\mathrm{A}$ or $\mathrm{B}$. Then

$$
\tau_{B}^{-1}|D| \subset|m H|
$$

for $m<n$.

Proof. Let $B^{*}$ be the line conjugate to $B$ with respect to the double cover morphism $\pi: V \rightarrow Q$. We set $\nu=\operatorname{mult}_{B}|D|(\nu>n$ by assumption $)$ and $\nu^{*}=\operatorname{mult}_{B^{*}}|D|$.

Suppose that $B$ is a line of type A. Then the proof of the analogous assertion in [1] carries over word for word to our situation. Furthermore,

$$
\tau_{B}^{-1}|D| \subset|(9 n-8 \nu) H|
$$

and it is clear that $9 n-8 \nu<n$.

Suppose now that $B$ is a line of type $\mathrm{B}, \varphi_{0}: V_{0} \rightarrow V$ is a blow-up of the singular point $P \in V, E_{0}$ is the exceptional divisor, $B^{0}=\varphi_{0}^{-1}(B)$, and $B^{* 0}=\varphi_{0}^{-1}\left(B^{*}\right)$; further, let $\psi: \bar{V} \rightarrow V_{0}$ be a successive blow-up first of $B^{0}$, and then of the proper inverse image $B^{* 0}$, and let $E=\psi^{-1}\left(B^{0}\right)$ and $E^{*}=\psi^{-1}\left(B^{* 0}\right)$.

We consider a section $Q_{H}$ of $Q$ by a general hyperplane in $\mathbb{P}^{4}$ that passes through $\pi(B), H=\pi^{*}\left(Q_{H}\right)$

$$
H^{0}=\varphi_{0}^{-1}(H)=H^{*}-E_{0},
$$

and

$$
\bar{H}=\psi^{-1}\left(H^{0}\right)=H^{*}-E_{0}^{*}-E-E^{*} .
$$

The linear system $|\bar{H}|$ gives a morphism $\varphi_{|\bar{H}|}: \bar{V} \rightarrow \mathbb{P}^{2}$ that realizes $\bar{V}$ as a fibring into curves of arithmetic genus one. The birational involution $\bar{\tau}_{B}$ is defined, if the fibre is irreducible, to be a fibrewise map with respect to $E^{*}$ (in the sense of the group law). Let $Q_{P}=Q \cap \overline{T_{P} Q}$. Despite the fact that a whole open subset of $\bar{E}_{0} \cup \bar{Q}_{P}$, where $\bar{E}_{0}=\psi^{-1}\left(E_{0}\right)$ and $\bar{Q}_{P}=\left(\pi \circ \varphi_{0} \circ \psi\right)^{-1}\left(Q_{P}\right)$, is composed of reducible fibres, there is an open set $U=\bar{V} \backslash\{$ a finite set of curves $\}$ on which $\bar{\tau}_{B}$ is biregular.

To see this it suffices to consider the restriction of $\bar{\tau}_{B}$ to the surface $\bar{S}$, where

$$
\bar{S}=\left(\pi \circ \varphi_{0} \circ \psi\right)^{-1}\left(Q_{L}\right)
$$

and $Q_{L}$ is the section of $Q$ by a general hyperplane $L \subset \mathbb{P}^{4}$, so that $Q_{L} \cap Q_{P}=l \cup l^{\prime}$, $l^{\prime}$ is a line passing through $P$, and $Q_{L}$ does not contain lines of type $\mathrm{A}$ or B other than $l$.

Under such conditions $\bar{S}$ is a non-singular K3 surface; the restriction of $\varphi_{|\bar{H}|}$ to $\bar{S}$ gives a morphism $\bar{g}: \bar{S} \rightarrow \mathbb{P}^{1}$, the curves $\bar{B}=E \cap \bar{S}$ and $\bar{B}^{*}=E^{*} \cap \bar{S}$ are sections of this morphism, and, moreover, $\psi(\bar{B})=B^{0}$ and $\psi\left(\bar{B}^{*}\right)=B^{* 0}$; finally, all the fibres of $\bar{g}$ except one are irreducible. The unique reducible fibre is $C_{1} \cup C_{2}$, where $\left(\pi \circ \varphi_{0} \circ \psi\right)\left(C_{1}\right)=l^{\prime}$ and $C_{2}=\bar{E}_{0} \cap \bar{S}$. We note that $\operatorname{Pic}(\bar{S})$ is generated by the classes $H_{S}^{*}=\left.H^{*}\right|_{\bar{S}}, \bar{B}, \bar{B}^{*}, C_{1}$, and $C_{2}$.

Obviously, every birational automorphism is biregular on $\bar{S}$ and hence $\left.\bar{\tau}_{B}\right|_{\bar{S}}$ extends to an automorphism of $\bar{S}$, and thus $\bar{\tau}_{B}$ is biregular on $\bar{V}$ outside codimension two. 
Our immediate problem is to define the action of $\bar{\tau}_{B}$ on $\operatorname{Pic}(\bar{V})$. Since $\bar{S}$ is invariant with respect to $\bar{\tau}_{B}$, it suffices to carry out the computations on $\operatorname{Pic}(\bar{S})$.

Let $X$ be the fibre of the morphism $\bar{g}: \bar{S} \rightarrow \mathbb{P}^{1}$. We have the following relations in $\operatorname{Pic}(\bar{S})$ :

$$
\begin{aligned}
& X^{2}=0 ; \\
& H_{S}^{* 2}=4 ; \\
& \bar{B}^{2}=\bar{B}^{* 2}=C_{1}^{2}=C_{2}^{2}=-2 ; \\
& \bar{B} \circ \bar{B}^{*}=\bar{B} \circ X=\bar{B} \circ C_{2}=\bar{B}^{*} \circ X=\bar{B}^{*} \circ C_{2}=1 ; \\
& \bar{B} \circ C_{1}=\bar{B}^{*} \circ C_{1}=X \circ C_{1}=X \circ C_{2}=0 ; \\
& C_{1} \circ C_{2}=2 .
\end{aligned}
$$

It is clear that $\left.\bar{\tau}_{B}\right|_{S}{ }^{*}(X)=X$ and $\left.\bar{\tau}_{B}\right|_{\bar{S}}{ }^{*}\left(\bar{B}^{*}\right)=\bar{B}^{*}$. Moreover, since $C_{1} \cup C_{2}$ is invariant with respect to $\left.\bar{\tau}_{B}\right|_{\bar{S}}, C_{1}+C_{2} \sim X, C_{1} \circ \bar{B}^{*}=0$, and $C_{2} \circ \bar{B}^{*}=1$, we have that $\left.\bar{\tau}_{B}\right|_{\bar{S}}{ }^{*}\left(C_{1}\right)=C_{1}$ and $\left.\bar{\tau}_{B}\right|_{\bar{S}}{ }^{*}\left(C_{2}\right)=C_{2}$.

This implies the following relations:

$$
\begin{aligned}
& \left.\left.\bar{\tau}_{B}\right|_{S} ^{*}(\bar{B}) \circ \bar{\tau}_{B}\right|_{\bar{S}} ^{*}(\bar{B})=-2 ; \\
& \left.\bar{\tau}_{B}\right|_{\bar{S}} ^{*}(\bar{B}) \circ X=1 ; \\
& \left.\bar{\tau}_{B}\right|_{\bar{S}} ^{*}(\bar{B}) \circ \bar{B}^{*}=1 ; \\
& \left.\bar{\tau}_{B}\right|_{\bar{S}} ^{*}(\bar{B}) \circ C_{1}=0 ; \\
& \left.\bar{\tau}_{B}\right|_{\bar{S}} ^{*}(\bar{B}) \circ C_{2}=1 .
\end{aligned}
$$

Let

$$
\left.\bar{\tau}_{B}\right|_{\bar{S}} ^{*}(\bar{B})=\alpha X+\beta \bar{B}+\gamma \bar{B}^{*}+\delta C_{1}+\varepsilon C_{2} .
$$

Then the relations (17) and (18) imply two possible sets of coefficients:

$$
\begin{aligned}
& \beta=1, \quad \gamma=0, \quad \varepsilon+\alpha=0, \quad \text { and } \quad \varepsilon=\delta ; \\
& \beta=-1, \quad \gamma=2, \quad \varepsilon+\alpha=6, \quad \text { and } \quad \varepsilon=\delta .
\end{aligned}
$$

We are not interested in the first case, because then $\left.\bar{\tau}_{B}\right|_{\bar{S}}=\left.\mathrm{id}\right|_{\bar{S}}$. In the second case, using the fact that $C_{1}+C_{2} \sim X$, we have that

$$
\begin{aligned}
\left.\bar{\tau}_{B}\right|_{\bar{S}} ^{*}(\bar{B}) & =6 H_{S}^{*}-7 \bar{B}-4 \bar{B}^{*}-6 C_{2} ; \\
\left.\bar{\tau}_{B}\right|_{\bar{S}} ^{*}\left(H_{S}^{*}\right) & =7 H_{S}^{*}-8 \bar{B}-4 \bar{B}^{*}-6 C_{2} .
\end{aligned}
$$

Thus, $\bar{\tau}_{B}$ acts on $\operatorname{Pic}(\bar{V})$ as follows:

$$
\begin{aligned}
\bar{\tau}_{B}^{*}\left(H^{*}\right) & =7 H^{*}-8 E-4 E^{*}-6 \bar{E}_{0} ; \\
\bar{\tau}_{B}^{*}(E) & =6 H^{*}-7 E-4 E^{*}-6 \bar{E}_{0} ; \\
\bar{\tau}_{B}^{*}\left(E^{*}\right) & =E^{*} ; \\
\bar{\tau}_{B}^{*}\left(\bar{E}_{0}\right) & =\bar{E}_{0} .
\end{aligned}
$$


Next, let $|\bar{D}|$ be the proper inverse image of $|D|$ on $\bar{V}$. By assumption,

$$
|\bar{D}| \subset\left|n H^{*}-\nu E-\nu^{*} E^{*}-\nu_{0} \bar{E}_{0}\right| .
$$

From the relations (20),

$$
\bar{\tau}_{B}^{-1}|\bar{D}| \subset\left|(7 n-6 \nu) H^{*}-(8 n-7 \nu) E-\left(4 n-4 \nu+\nu^{*}\right) E^{*}-\nu_{0} \bar{E}_{0}\right| .
$$

Finally, lowering all this to $V$, we see that $\tau_{B}^{-1}|D| \subset|(7 n-6 \nu) H|$ and $7 n-6 \nu<n$. The proposition is proved.

\section{$\S 6$. Completion of the proof}

The last thing remaining for us in the proof of the assertions formulated in $\S 1$ is to see that there are no relations in the group $B(V) \subset \operatorname{Bir}(V)$ generated by all the birational involutions of the form $\tau_{B}$. Indeed, the arguments of the preceding section give us the existence of an exact triple

$$
1 \longrightarrow B(V) \longrightarrow \operatorname{Bir}(V) \longrightarrow \operatorname{Aut}(V) \longrightarrow 1 .
$$

If we now prove that the linear system $|D|$ cannot simultaneously have two maximal singularities, then we thereby prove the uniqueness of the process of 'untwisting' any birational automorphism (that is, the uniqueness of the representation

$$
\chi=g \circ \tau_{B_{1}} \circ \cdots \circ \tau_{B_{k}},
$$

where $g \in \operatorname{Aut}(V))$, from which it will follow that $B(V)$ is a free product of birational involutions.

Suppose that the linear system $|D|$ has maximal singularities at the lines $B_{1}$ and $B_{2}$; we can single out four cases of their mutual arrangement:

a) $B_{1} \neq B_{2}, B_{1} \cap B_{2}=\varnothing$;

b) $B_{1} \neq B_{2}, B_{1} \cap B_{2}=$ a point, $B_{1} \neq \delta\left(B_{2}\right)$, where $\delta$ is the double cover involution;

c) $B_{1} \neq B_{2}, B_{1}=\delta\left(B_{2}\right)$;

d) $B_{1}=B_{2}$, that is, $B_{2}$ lies 'over' $B_{1}$ (the centre of an infinitely near singularity).

All this is actually analyzed in [1]: the cases a) and d), along with b) and c) under the condition that the intersection point is not singular, carry over in general without changes to our situation, and the remaining two cases we consider may differ at most by the coefficients in the formulae.

Case b). Suppose that $B_{1} \cap B_{2}=\{$ a singular point $\}$. We consider a linear subsystem $|S| \subset|2 H|$ on $|V|$ such that Bas $|S| \cap \operatorname{Bas}|D|=B_{1} \cup B_{2} \cup$ isolated points\}; the fact that it is non-empty is ensured by the Riemann-Roch theorem $(|S|$ is actually concerned with separating the fibres of the cover $\pi: V \rightarrow Q$ ). We set $\nu_{i}=$ mult $_{i}|D|>n, i=1,2$, and let $\psi: \bar{V} \rightarrow V$ be the composition of the blow-ups first of the singular point, and second of the proper inverse images of $B_{1}$ and $B_{2}$. Then for the proper inverse images on $\bar{V}$ we have that $\bar{D}^{2} \circ \bar{S} \geqslant 0$. On the other hand, it is not hard to compute that

$$
\bar{D}^{2} \circ \bar{S}=8 n^{2}-2 \nu_{0}^{2}-3 \nu_{1}^{2}-3 \nu_{2}^{2}-2\left(n-\nu_{0}\right)\left(\nu_{1}+\nu_{2}\right)<0
$$


for $0 \leqslant \nu_{0} \leqslant n$. We have obtained a contradiction.

Case c). Suppose that the line $\pi\left(B_{1}\right)=\pi\left(B_{2}\right)$ passes through the singular point on the branch divisor, $S$ is the proper inverse image of a general hyperplane section of the quadric $Q$ through this line, and $\psi: \bar{V} \rightarrow V$ is a composition of blow-ups as above. A straightforward computation again gives us that

$$
\bar{D}^{2} \circ \bar{S}=4 n^{2}-2 \nu_{0}^{2}-\nu_{1}^{2}-\nu_{2}^{2}-2\left(n-\nu_{0}\right)\left(\nu_{1}+\nu_{2}\right)-\left(\nu_{1}-\nu_{2}\right)^{2}<0
$$

for $0 \leqslant \nu_{0} \leqslant n$, and this contradicts the requirement $\bar{D}^{2} \circ \bar{S} \geqslant 0$.

The proof of the theorem in $\S 1$ is complete.

\section{REFERENCES}

1. V. A. Iskovskikh, "Birational automorphisms of three-dimensional algebraic varieties", Itogi Nauki i Tekhniki: Sovrem. Probl. Mat., vol. 12, VINITI, Moscow 1979, pp. 159-236; English transl. in J. Soviet Math. 13:6 (1980).

2. V. A. Iskovskikh and Yu. A. Manin, "Three-dimensional quartics and counterexamples to the Lüroth problem", Mat. Sb. 86 (128) (1971), 140-166; English transl. in Math. USSR-Sb. 15 (1971).

3. A. V. Pukhlikov, "Birational automorphisms of a three-dimensional quartic with an elementary singularity", Mat. Sb. 135 (177) (1988), 472-496; English transl. in Math. USSR-Sb. 63 (1989).

4. A. V. Pukhlikov, "Birational automorphisms of a double space and a double quadric", Izv. Akad. Nauk SSSR Ser. Mat. 52 (1988), 229-239; English transl. in Math. USSR-Izv. 32 (1989).

5. A. V. Pukhlikov, "Essentials of the method of maximal singularities", University of Warwick Preprints: $31 / 1996$.

Steklov Institute of Mathematics

Russian ACAdemy of Sciences

E-mail address: grin@ mi.ras.ru 